Accelerator Division

Alternating Gradient Synchrotron Department

BROOKHAVEN NATIONAL LABORATORY

Upton, New York 11973

Accelerator Division

Technical Note

AGS/AD/Tech. Note No. 363

\title{
THE DESIGN OF THE DIPOLE PICK-UP COILS FOR USE WITH THE AGS GAUSS CLOCK
}

\author{
A. Warner and W. VanZwienen \\ October 7, 1992
}

\section{Introduction}

The pick-up coils for the new AGS gauss clock consist of four coils, wound on two cores (two coils on each core). The redundant system allows simultaneous running of gauss clocks. The gauss clock coils will be installed into magnet \# 242 which is located in the basement of the Siemens building. Magnet \# 242 is a "long" open magnet, (i.e., the length of the magnet steel is 90 inches). The coils will provide a voltage which is proportional to the rate of change of magnetic flux $(\dot{B})$. The coil windings and the form dimensions have been chosen to produce approximately 5 Volts $\backslash 3$ Teslalsec. This note describes the design of the coils and documents some of the major mechanical design considerations.

Summary of AGS Dipoles

The AGS lattice includes 240 dipole magnets which are classified in the following way: ${ }^{1}$

\section{Type}
A (open)
$B$ (open)
C (closed)

Number of Magnets

48 (long)

96 (short)

96 (long)
Length of Magnet

90 inches

75 inches

90 inches

Type A and B magnets are opened faced (see Fig. A1) and type C are closed. In addition to this, the magnets are classified as either long or short depending on whether they are 90 or 75 inches. The design of the new gauss clock accounts for this difference in magnetic length around the ring by taking the average of all the magnets in the ring. To accomplish this, two 
cores are placed in the reference magnet \# 242 at a predetermined distance from each end of the magnet leaving a gap of approximately 2 inches in the middle. This gap accounts for the discrepancy in the magnetic length integrated over the ring (i.e. average).

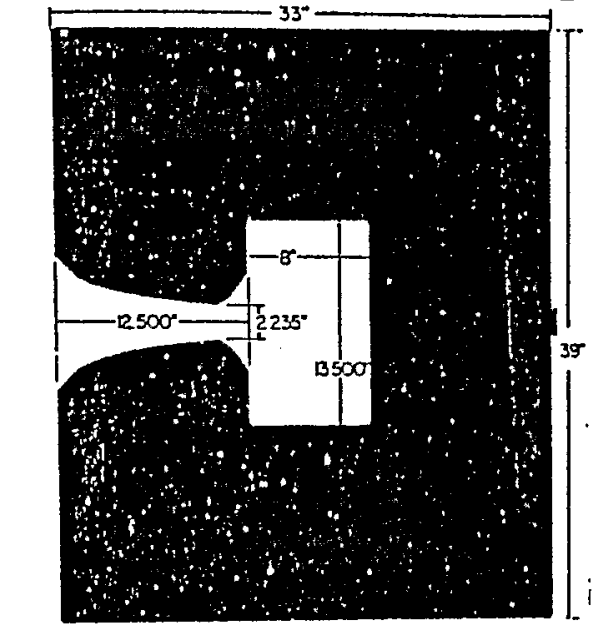

Figure A1: An "open" magnet lamination, showing cross-section of a typical AGS magnet.

The dimensions of the core are chosen to maximize the signal and to support two coils; an inner coil of 54 turns and an outer coil of 52 turns nested within the first coil. The dimensions of this arrangement is shown in Figures 1-5. All coils are made of \# 30 wire (12 mil diameter). The signal from the coils are electrically summed and are dependent on $\dot{B}$ and the coil characteristics as follows:

$$
V_{d i p o l e}=\frac{d B}{d t} x W x N x L
$$

where:

$$
\begin{array}{ll}
\mathrm{A}_{\mathrm{cff}} & =\text { Effective Area }=\mathrm{WxNxL} \\
\mathrm{W} & =\text { coil width }(\mathrm{cm}) \\
\mathrm{N} & =\# \text { of turns } \\
\mathrm{L} & =\text { length of coil }(\mathrm{cm}) \\
\frac{\mathrm{dB}}{\mathrm{dt}} & =\dot{B}(\text { Tesla/sec) }
\end{array}
$$

The inner and outer coils were designed to have an effective area of $16817.31 \mathrm{~cm}^{2}$ and $16570.19 \mathrm{~cm}^{2}$ respectively. The emf generated with these characteristics are 5.045 volts and 4.975 volts after scaling to $5 \mathrm{volt} / 3 \mathrm{Tesla} / \mathrm{sec}$.

\section{Errors due to rotation and lateral motion of the coils}

Error due to rotation of one coil about an axis through its center. It is possible that one coil or even both coils are rotated in some way. This would introduce a cosine dependent component into the coil. For example:

$$
\mathrm{B}_{\mathrm{actual}}=\mathrm{B} \operatorname{Cos} \theta
$$




$$
\begin{gathered}
\operatorname{Cos} \theta=1-\frac{\theta^{2}}{2}+\frac{\theta^{4}}{4}-\ldots \\
\text { relative error }=\frac{B-B_{\text {actual }}}{B_{\text {actual }}}=\frac{B-B \cos \theta}{B \cos \theta}=\frac{\left[\frac{2 \pi \theta}{360}\right]^{2}}{2}
\end{gathered}
$$

$\rightarrow 1.52 \times 10^{-4}$ relative error for $1^{\circ}$ rotation.

\section{Error Due to Radial Position}

The gradient in the AGS dipole magnets is $10 \%$ per inch. Therefore, an error of $0.1 \mathrm{mil}$ in the radial position of the coil could introduce a relative error of $1 \times 10^{-5}$ in the system.

\section{Major Mechanical Design Considerations}

One design requirement was that all components except for the coil and vacuum chamber surrounding the coil be made of non-metallic, low thermal expansion material. A fiberglass material manufactured under the trade name of Extren* was used for critical components such as the coil core, the support cradle and other components. Marble was also considered but it is not cost effective since it is ten times as expensive and can be easily broken during the machining process. Extren also has the added advantage that it is available in lengths of 20 feet and in various shapes. The coil support cradle is ten feet long and therefore use of Extren did not require splicing. However, machining of Extren is more troublesome than that of G10 which,unfortunately, is not manufactured in length of more than eight feet.

The longitudinal axis of the coils must be located to an accuracy of .010 inches of the magnet aperature axis which corresponds to the beam centerline in order to achieve gauss clock accuracy of one part in $10^{4}$. Also required, is that following removal of the coil, cradle and surrounding chambers from the magnet, for any reason, they must be reinstallable within a few thousandths of an inch of its original position without the need of adjustment. To satisfy this requirement the support components must be machined to close tolerances.

* Morrison Molder Fibre Glass Co., Bristol, VA. 24203 
Due to the fact the upper pole of the magnets move towards the lower pole by about $0.015^{\prime \prime} / 13 \mathrm{~kg}$ each time the magnets are pulsed, it was decided not to support the coil with fixtures that contacted both poles. Instead, the coil as well as the surrounding chamber is supported by the lower pole section only. This allows the upper pole section to move during pulsing without influencing the coil or chamber positions in space.

Both the coil and the surrounding inconel chamber must be accurately located in the reference magnet while at the same time being separated from each other. They are independently mounted so that the motion of the chamber does not affect the coil location.

The Fundamental Idea of the Gauss Clock Measurement

The gauss clock measures the integrated value of $\dot{B}$ evaluated from some start time " $\mathrm{t}_{0}$ " to some final time " $t$ ", it is, therefore, not an absolute measurement of the magnetic field in the machine. For example:

$$
B(t)=B_{o}\left(t_{o}\right)+\int_{t_{o}}^{t_{1}} \frac{d B}{d \tau} \mid \tau d \tau
$$

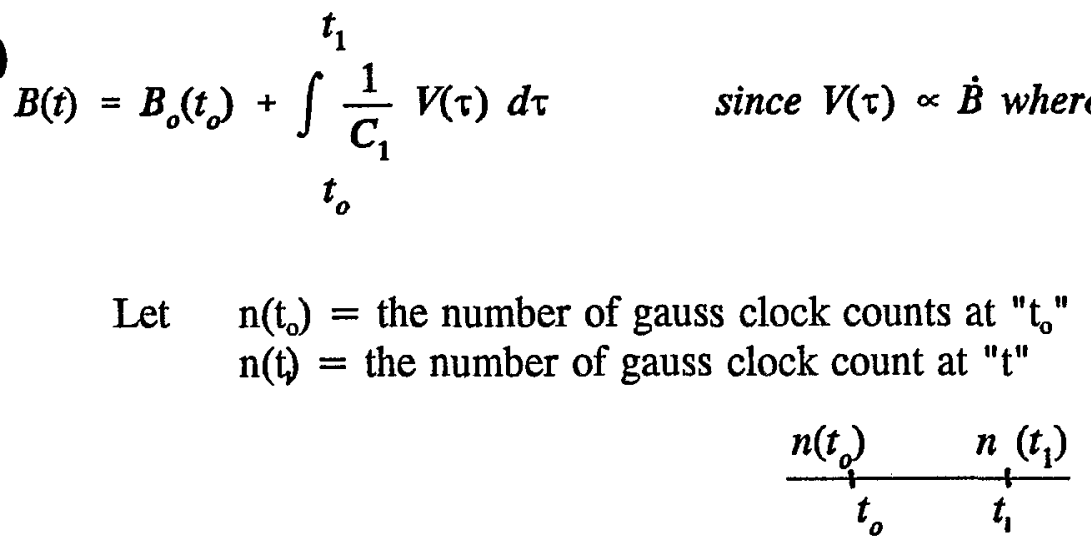

then the frequency $f=\varliminf_{t_{0} \rightarrow t_{1}} \frac{n\left(t_{1}\right)-n\left(t_{0}\right)=\frac{d n}{d t}}{t_{1}-t_{o}}$ 


$$
\begin{gathered}
B(t)=B\left(t_{0}\right)+\frac{1}{c_{1}} \int_{t_{o}}^{t_{1}} d \tau \frac{1}{c_{2}} f(\tau) \quad \text { where } \frac{1}{c_{2}} \text { is the known const relating } f \text { and } \dot{B} \\
=B\left(t_{0}\right)+\left.\frac{1}{C_{3}} \int^{t} d \tau \frac{d n}{d t}\right|_{\tau} \text { where } \frac{1}{c_{3}} \text { is the combined const } \frac{1}{c_{1}} \text { and } \frac{1}{c_{2}} \\
t_{0} \\
B(t)=B\left(t_{0}\right)+\frac{1}{c_{3}}\left(n\left(t_{1}\right)-n\left(t_{o}\right)\right) \\
=B\left(t_{0}\right)+\frac{1}{c_{3}} \Delta n\left(t_{1}-t_{o}\right)
\end{gathered}
$$

\section{Conclusion:}

The hardware electronics of the AGS gauss clock will be similar to that of the Booster gauss clock. ${ }^{2}$ The coils have been designed to measure the field that the beam will see as accurately as possible. Care has been taken to reduce errors due to motion of the coils and differences in the magnets.

\section{References:}

1. R.A. Beth and C. Lasky, The Brookhaven AGS Science, December 5, 1958, Vol. 128, No. 3336, pgs. 1393-1401.

2. J.Geller, A Digital Voltage to Frequency Convertor for the Booster Gauss Clock, Booster Tech. Note \# 175, July 25, 1990. 
Figure 1: A sketch of the gauss clock general arrangement including data related to the coil.

Figure 2: Shows the major components that make up the gauss clock installed into an AGS magnet. (The actual magnet to be used is a long open one located in the basement of the Siemens Bldg.)

Figure 3: Shows end view A-A of Fig. 2 which illustrates the support means for the extreme outboard ends of the cradle which serves as the support beam for the two coils.

Figure 4: Shows section B-B of Fig. 2 which is typical at two locations. It illustrates the means used to support and locate the inconel chamber independent of the cradle and coils.

Figure 5: Shows section C-C of Fig. 2 which is typical at three locations. It illustrates the method of supporting the coil and cradle independent from the surrounding chamber. Two pins at each of the three locations provide support and location for the cradle and coil which is attached to the cradle. The pins pass through clearance holes in the chamber and therefore have no contact with the chamber. 

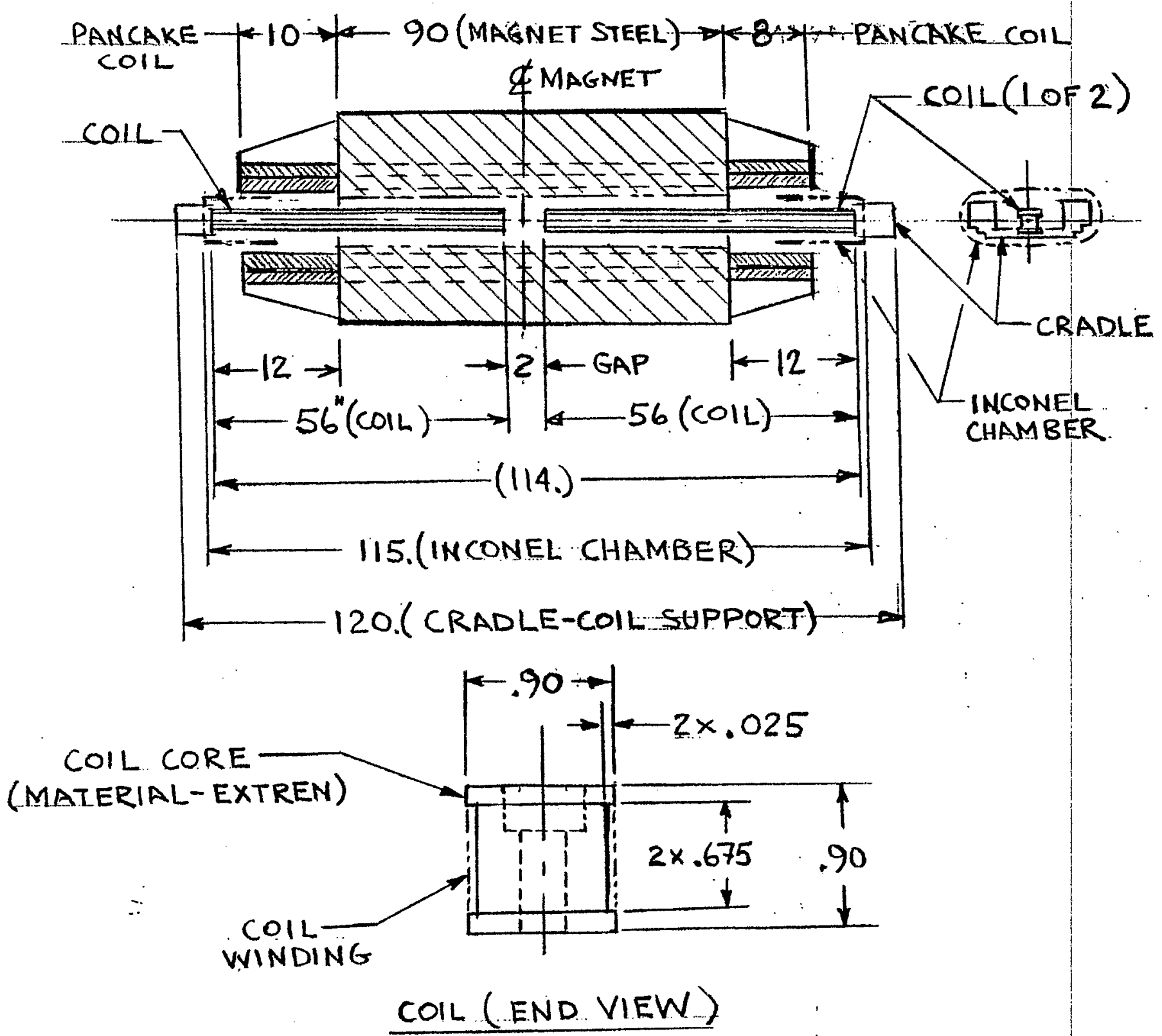

NUMBER OF TURNS (OUTER COIL) 52

NUMBER OF TURNS (INNER COIL) .54

$\left.\begin{array}{l}\text { SCALED VOLTAGE (OUTER COIL) } 4.975 \text { VOLTS } \\ \text { SCALED VOLTAGE (INNER COIL) } 5.045 \text { VOLTS }\end{array}\right\}$

FIGURE 1

GENERAL ARRANGEMENT SKETCH AND COIL DATA

(SCALE: NONE; DIMENSIONS IN INCHES) 


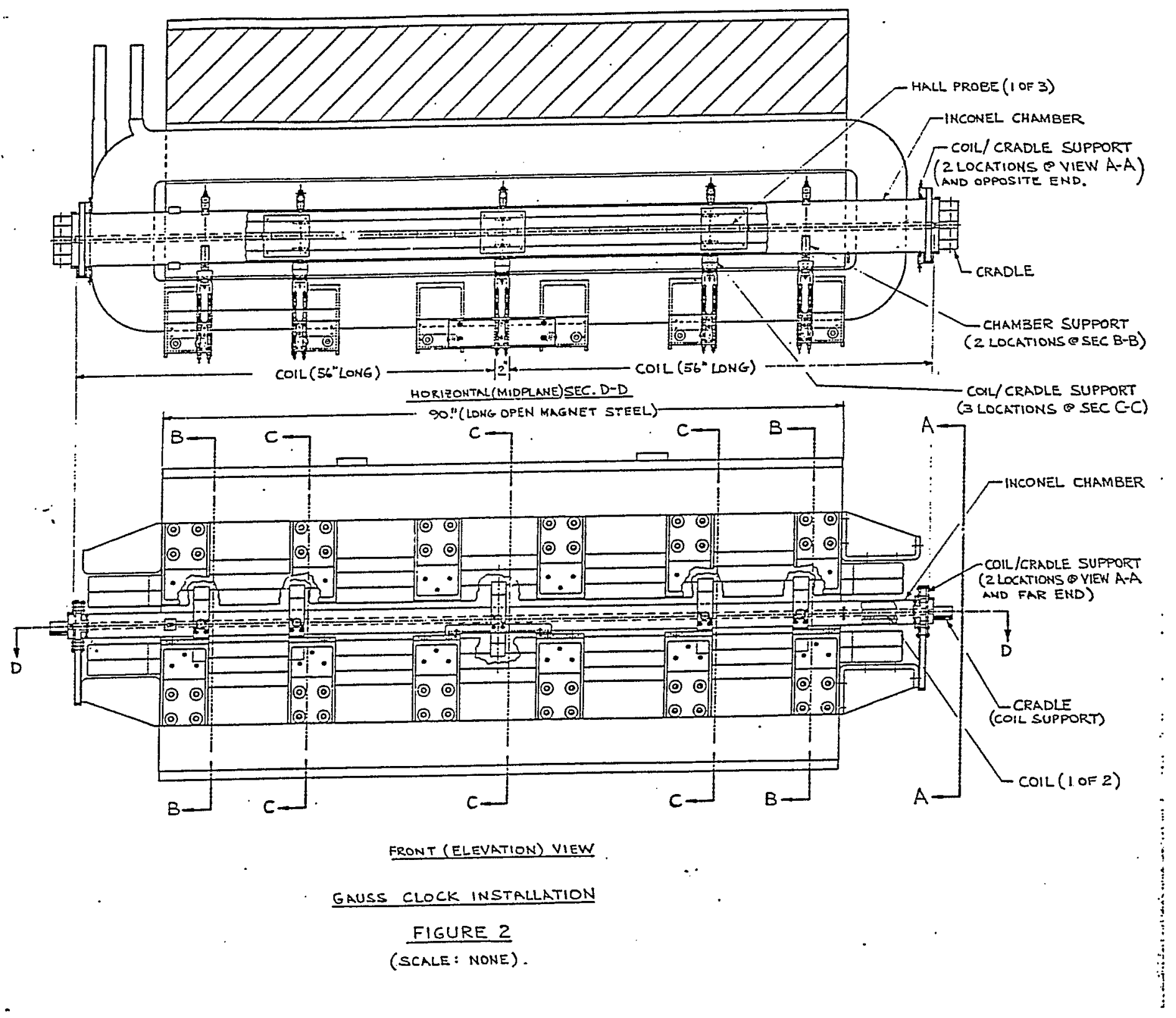


INCONEL CHAMBER-

CLEARANCE (.020"APPROX.)

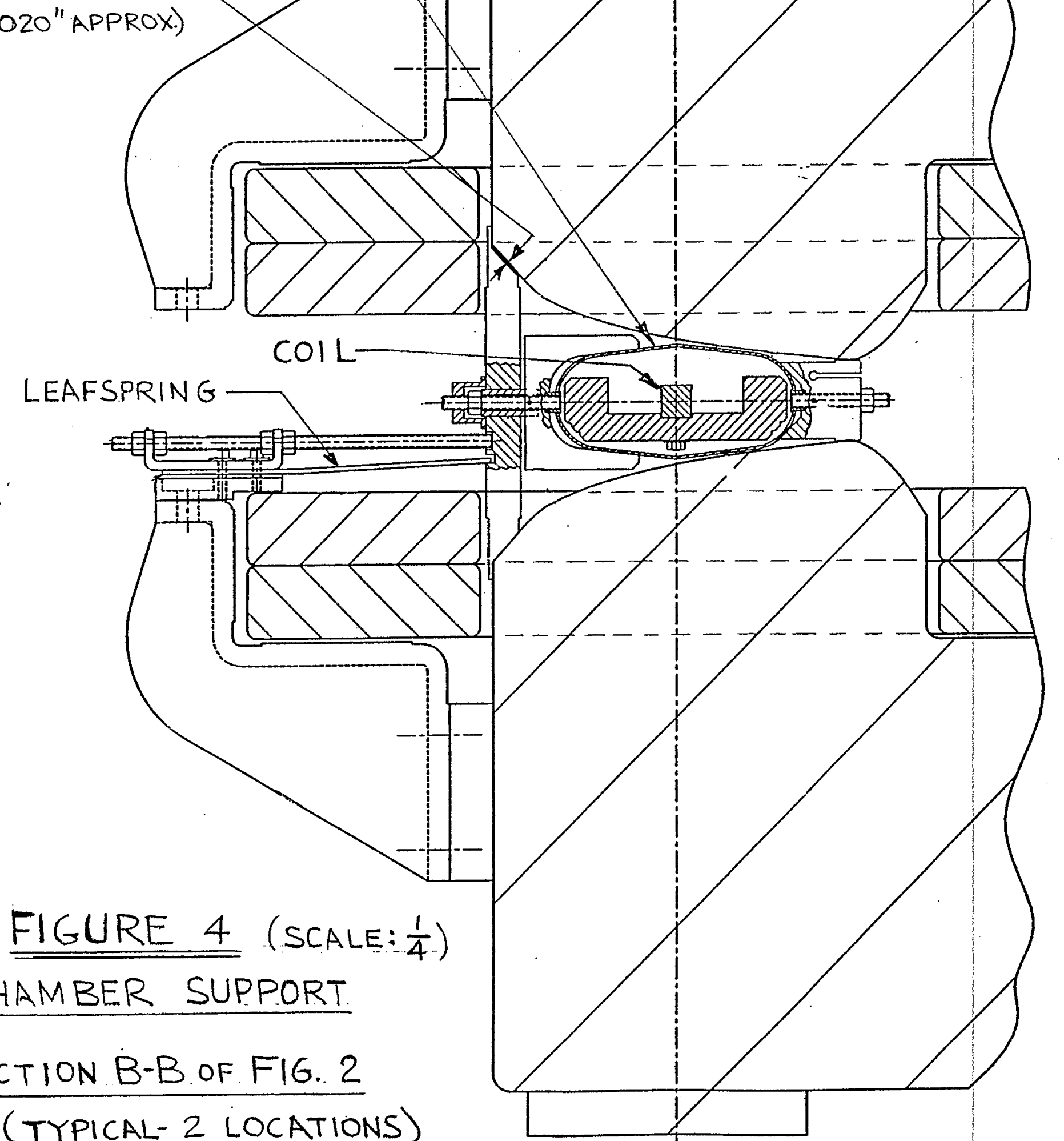


HALL PROBE (I OF 3) INCONEL CHAMBER-

CLEARANCE

.020 "APPROX.)

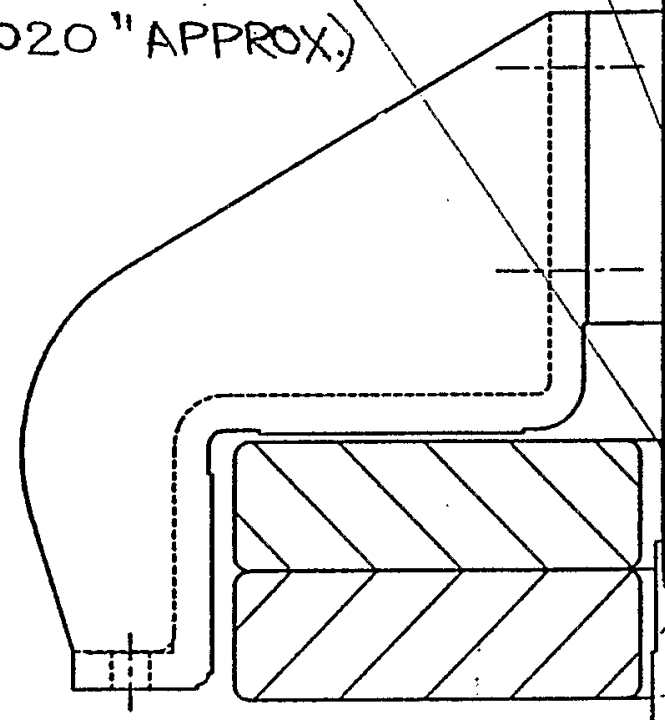

\section{$\mathrm{COIL}$}

LEAFSPRING
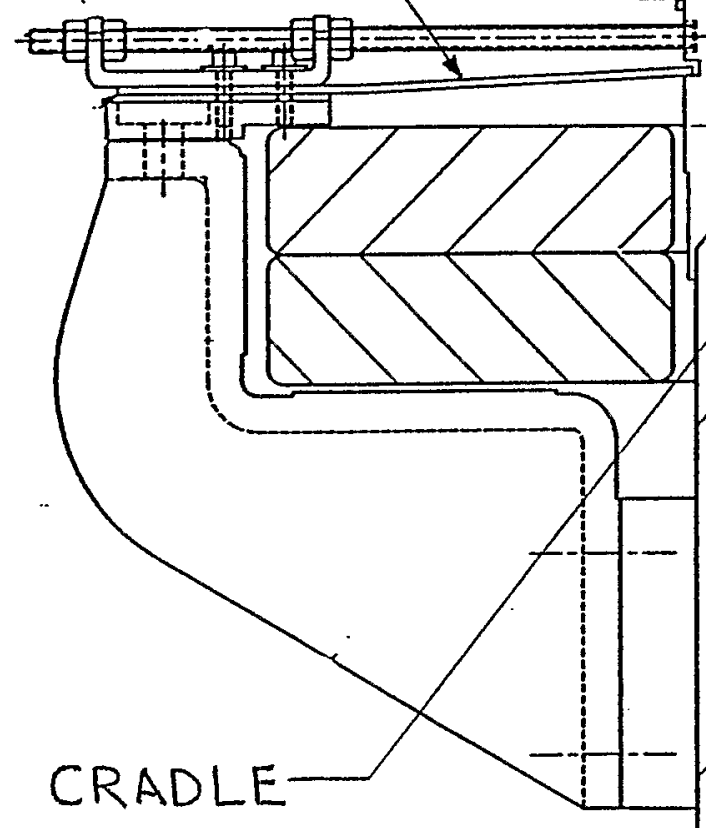

FIGURE 5 (SCALE: $\frac{1}{4}$ )

DIL/CRADLE SUPPORT

SECTION C-C OF FIG. 2

(TYPICAL-3 LOCATIONS) 